\title{
A 10-h period revealed in optical spectra of the highly variable WN8 Wolf-Rayet star WR 123 ${ }^{\star, \star \star}$ (Research Note)
}

\author{
A.-N. Chené ${ }^{1,2,3}$, C. Foellmi ${ }^{4}$, S. V. Marchenko ${ }^{5}$, N. St-Louis ${ }^{6}$, A. F. J. Moffat ${ }^{6}$, D. Ballereau ${ }^{7}$, J. Chauville ${ }^{7}$, \\ J. Zorec ${ }^{8}$, and C. A. Poteet ${ }^{9}$ \\ ${ }^{1}$ Departamento de Astronomía, Casilla 160-C, Universidad de Concepción, Chile \\ e-mail: achene@astro-udec.cl \\ 2 Departamento de Física y Astronomía, Facultad de Ciencias, Universidad de Valparaíso, Av. Gran Bretaña 1111, Playa Ancha, \\ Casilla 5030 Valparaíso, Chile \\ 3 Canadian Gemini Office, Herzberg Institute of Astrophysics, 5071, West Saanich Road, Victoria (BC), V9E 2E7, Canada \\ 417 Bd Agutte Sembat, 38000 Grenoble, France \\ e-mail: cedric. foellmi@gmail.com \\ 5 Science Systems and Applications, Inc., 10210 Greenbelt Road, Suite 600, Lanham, MD 20706, USA \\ e-mail: sergey.marchenko@ssaihq.com \\ ${ }^{6}$ Département de Physique and CRAQ, Université de Montréal, C.P. 6128, Succ. Centre-Ville, Montréal, Québec, H3C 3J7, Canada \\ e-mail: [stlouis; moffat]@astro.umontreal.ca \\ 7 GEPI, UMR 8111 du CNRS, Observatoire de Paris-Meudon, 92195 Meudon, France \\ e-mail: [dominique.ballereau; jacques.chauville]@obspm.fr \\ ${ }^{8}$ Institut d'Astrophysique de Paris, UMR 7095 CNRS-Université Pierre \& Marie Curie, 98bis, BD. Arago, 75014 Paris, France \\ e-mail: zorec@iap.fr \\ 9 Ritter Observatory, Department of Physics \& Astronomy, The University of Toledo, Mail Stop \#113, Toledo, Ohio 43606-3390, \\ USA \\ e-mail: cpoteet@physics.utoledo.edu
}

Received 24 January 2011 / Accepted 11 April 2011

\section{ABSTRACT}

\begin{abstract}
Aims. What is the origin of the large-amplitude variability in Wolf-Rayet WN8 stars in general and WR123 in particular? A dedicated spectroscopic campaign targets the ten-hour period previously found in the high-precision photometric data obtained by the MOST satellite.

Methods. In June-August 2003 we obtained a series of high signal-to-noise, mid-resolution spectra from several sites in the $\lambda \lambda$ 4000-6940 Å domain. We also followed the star with occasional broadband (Johnson $V$ ) photometry. The acquired spectroscopy allowed a detailed study of spectral variability on timescales from $\sim 5$ min to months.

Results. We find that all observed spectral lines of a given chemical element tend to show similar variations and that there is a good correlation between the lines of different elements, without any significant time delays, save the strong absorption components of the HeI lines, which tend to vary differently from the emission parts. We find a single sustained periodicity, $P \sim 9.8 \mathrm{~h}$, which is likely related to the relatively stable pulsations found in MOST photometry obtained one year later. In addition, seemingly stochastic, large-amplitude variations are also seen in all spectral lines on timescales of several hours to several days.
\end{abstract}

Key words. stars: individual: HD 177230 (WR 123) - stars: Wolf-Rayet - stars: winds, outflows - stars: oscillations stars: variables: general

\section{Introduction}

Population I Wolf-Rayet (WR) stars of the WN8 subclass have been the subject of numerous studies owing to their "enigmatic"

* Based on observations obtained at the Canada-France-Hawaii Telescope (CFHT) which is operated by the National Research Concil of Canada, the Institut National des Sciences de l'Univers of the Centre National de Recherche Scientifique of France, and the University of Hawaii. Based also on observations made with ESO Telescopes at the La Silla Observatory, under programme ID 271.D-5025.

$\star \star$ Photometric data presented in Fig. 1 are only available at the CDS via anonymous ftp to cdsarc.u-strasbg.fr (130.79.128.5) or via

http://cdsarc.u-strasbg.fr/viz-bin/qcat?J/A+A/530/A151 behavior. These direct descendants of massive and hot Of stars are markedly distinct from other WR stars. Indeed, no member of this class is known to have a close massive O-type companion. They avoid star clusters and associations. Their runaway frequency is relatively high, and in some cases their distance from the Galactic plane is large (Moffat \& Isserstedt 1980; Moffat 1989; Moffat et al. 1998). In addition, all photometric, polarimetric and spectroscopic monitoring campaigns of WN8 stars have consistently shown the highest level of intrinsic variability among all WR stars (e.g.; Moffat \& Shara 1986; Lamontagne \& Moffat 1987; Drissen et al. 1987; Balona et al. 1989; Robert et al. 1989; Robert 1992; Antokhin et al. 1995; Marchenko et al. 1998). However, despite the claims of multiple photometric periods ranging from hours to days, there is no period that could 
Table 1. Spectroscopic observing campaigns.

\begin{tabular}{lcccc}
\hline \hline & $\begin{array}{c}\text { La Silla } \\
\text { Observatory }\end{array}$ & $\begin{array}{c}\text { Observatoire de } \\
\text { Haute-Provence }\end{array}$ & $\begin{array}{c}\text { Canada-France- } \\
\text { Hawaii Telescope }\end{array}$ & $\begin{array}{c}\text { Observatoire du } \\
\text { mont Mégantic }\end{array}$ \\
\hline HJD $(-2452800)$ & $15.7,40.8-45.8$ & $28.5-34.5$ & $60.9-64.8$ & 73.6 \\
Telescope & $3.58 \mathrm{~m}(\mathrm{NTT})$ & $1.52 \mathrm{~m}$ & $3.6 \mathrm{~m}$ & $1.6 \mathrm{~m}$ \\
Instrument & EMMI & Aurélie & OSIS & OMM-Spectro \\
No. of spectra & 290 & 66 & 17 & 2 \\
Resolution, $\AA$ & $5.1(6$ pix $)$ & $1.4(3 \mathrm{pix})$ & $1.5(3$ pix $)$ & $1.6(3$ pix $)$ \\
Spectral range, $\AA$ & $4000-6940$ & $5225-6100$ & $4400-6100$ & $4500-6100$ \\
Signal-to-noise & $\geq 200$ & 100 & 200 & 100 \\
\hline
\end{tabular}

be labeled as unique to all the campaigns for any given WN8 star. Discovery of any stable periodicity pattern in a WN8 star would help to establish whether the star is a binary or a single rotator with an inhomogeneous wind or, alternatively, to refine the rather controversial pulsation models of WR stars.

All of the above applies specifically to our target, WR 123 (see Marchenko et al. 1998, for references), which shows an extremely complex, rapidly evolving light curve, along with seemingly aperiodic polarimetric (St-Louis et al. 1988) and high-amplitude line-profile variability (Massey \& Conti 1980; Lamontagne et al. 1983; Marchenko et al. 1998). All searches for periodic signals provided rather inconsistent results until 2004, when an intense, long-term, broad-band photometry campaign of WR 123 (Lefèvre et al. 2005) was performed by the MOST (Microvariability and Oscillations of STars) satellite. It was shown that large-scale, short-lived (typically, less than 1 week) photometric fluctuations were dominated by the evervariable continuum. Only one relatively stable, small-amplitude period was present in the entire data set at $P \approx 9.8 \mathrm{~h}$. Does this period indicate the detection of a second spectroscopic binary system among WN8 stars ${ }^{1}$ or does it provide a reliable observation of periodic stellar activity in a WR wind, like pulsations or co-rotating density structures?

While broadband photometry documents the variability of the (mainly) stellar continuum emanating from the inner WR wind, spectroscopy probes the layers outside it. Moreover, since the emission lines of different chemical species and/or different ionization potentials are generally formed in different regions of the wind, the timing and correlation of profile variations detected in such lines may help for determining how perturbations propagate in a stratified wind. In this paper we present the results of intense spectroscopic monitoring of WR 123.

\section{Observations and data reduction}

Originally, we planned to obtain spectroscopic monitoring in parallel with the MOST run, initially scheduled for June/July 2003. Therefore, we carried out spectroscopic observations of WR 123 during 5 runs on 4 different telescopes (Table 1). Unfortunately, the launch of MOST was delayed by one year. A small-scale spectroscopic campaign run in parallel with the 2004 MOST observations proved to be of critical importance and provided a picture, though rather sketchy, of line-profile variability (lpv) superposed on an ever-changing continuum flux (Lefèvre et al. 2005). Here, working with a unique, much more extensive data-set, we intend to obtain a more detailed description of the lpv, albeit outside the MOST campaign. The

\footnotetext{
1 The runaway WR148 = HD197406 is the first detected WN8 spectroscopic binary with a moderate-mass, unseen companion (Marchenko et al. 1996)
}

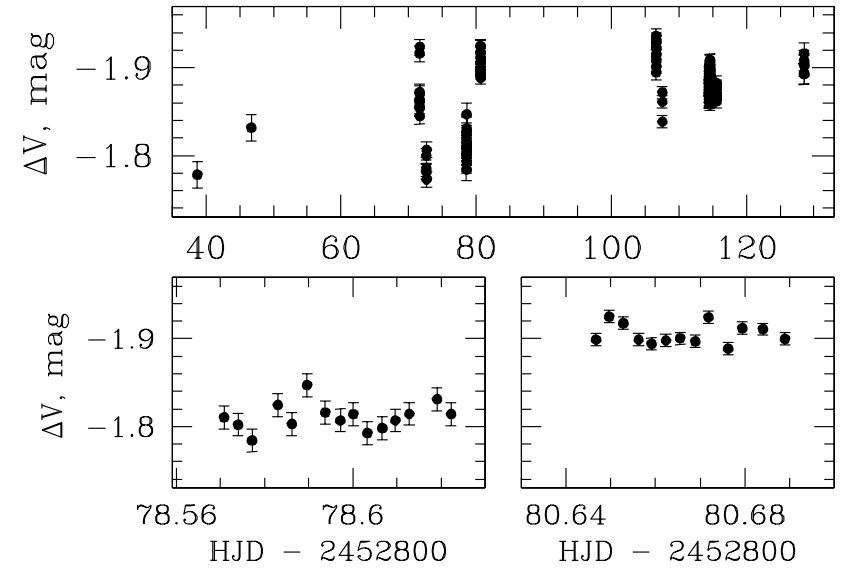

Fig. 1. The contemporaneous broad-band (Johnson $V$ ) photometry of WR 123 obtained at the Bell Observatory. $\Delta V$ is the difference between the magnitudes of WR 123 and comparison stars (see text). Each measurement is plotted with corresponding $\pm 1 \sigma$ error bars. The upper panel shows all acquired data while the lower panel depicts two nights from August 2003.

acquired spectroscopy allowed us to follow lpvs on timescales from $\sim 5$ min (NTT spectra only) to months (all spectra).

In parallel with the 2003 spectroscopic monitoring, we also performed broadband (Johnson V) photometry using the 24-inch telescope of the Bell Observatory (Western Kentucky University, USA). The differential photometry was carried out using the weighted combination of comparison stars in the $4.4 \times 6.6$ arcmin CCD field. We used comparisons with the following (epoch 2000) RA and Dec coordinates: $(\alpha=19: 03: 58.2$; $\delta=-04: 18: 48),(\alpha=19: 04: 04.6 ; \delta=-04: 17: 35),(\alpha=$ 19:03:45.3; $\delta=-04: 19: 30),(\alpha=19: 03: 54.7 ; \delta=-04: 20: 48)$, and 1.0:0.75:0.25:0.25 weights, as well as a star at $(\alpha=$ $19: 04: 13.2 ; \delta=-04: 20: 31)$ as a control star. We plot the available photometric data and corresponding $\pm 1 \sigma$ errors in Fig. 1 . All the spectroscopic and photometric data were processed using standard $\mathrm{IRAF}^{2}$ software packages. Special care was taken for the normalization of the spectra. First, a mean was made for each run. Then each spectrum of a run was divided by the run mean and the ratio fitted with a low order Legendre polynomial (between 4th and 8th order). The original individual spectrum was divided by this fit, and was therefore at the same level as the run mean. When this procedure was done for each run, the run means were then put at the same level by using the same procedure as described above. This allowed us to put all individual spectra at the same level. Then, we combined all run means

\footnotetext{
2 IRAF is distributed by the National Optical Astronomy Observatory, which is operated by the Association of Universities for Research in Astronomy, Inc. under cooperative agreement with the National Science Foundation.
} 


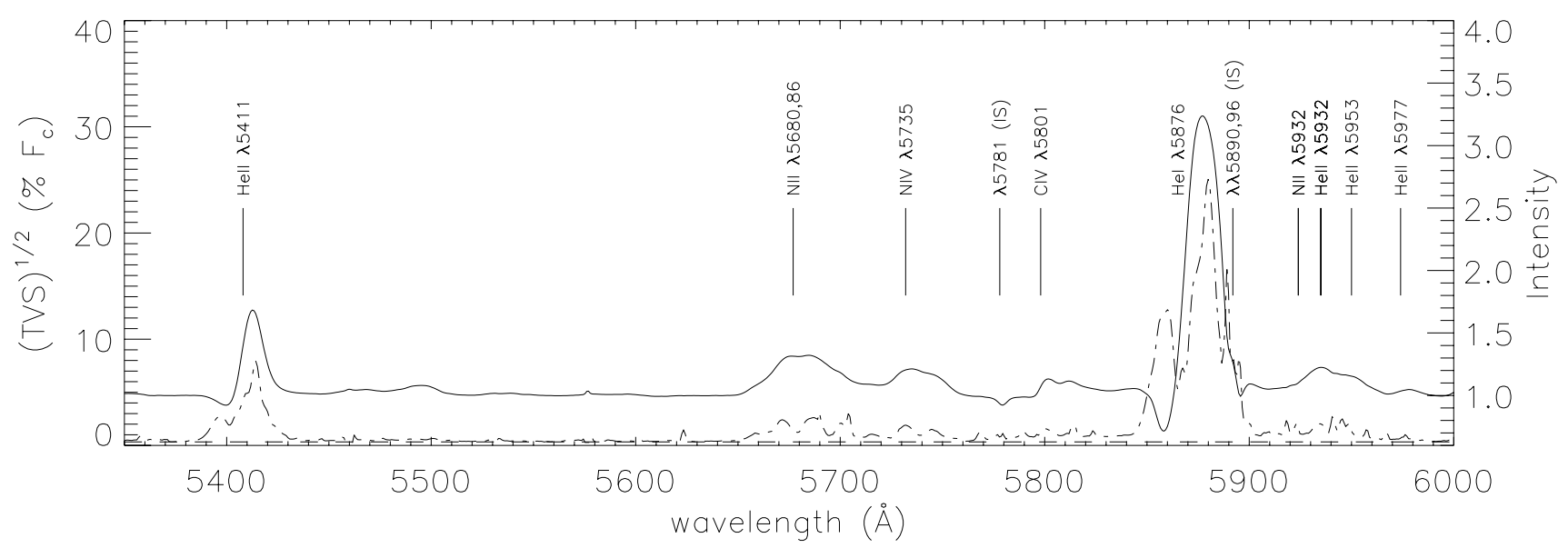

Fig. 2. Mean rectified spectrum (solid line) of WR 123 calculated using all the spectra from all the observing runs between $5350 \AA$ and $6000 \AA$ spectral range (common to all runs). The TVS spectrum (dash-dotted line) obtained using the formalism of Fullerton et al. (1996) is overplotted. The dashed line is the threshold of significant variability at a level of $99 \%$ for the TVS.

Table 2. Wavelength intervals of the selected pseudo-continuum regions.

\begin{tabular}{lcccc}
\hline \hline $\begin{array}{l}\text { Wav. int. } \\
(\AA)\end{array}$ & $\begin{array}{c}\text { Wav. int. } \\
(\AA)\end{array}$ & $\begin{array}{c}\text { Wav. int. } \\
(\AA)\end{array}$ & $\begin{array}{c}\text { Wav. int. } \\
(\AA)\end{array}$ & $\begin{array}{c}\text { Wav. int. } \\
(\AA)\end{array}$ \\
\hline $4001-4006$ & $4495-4498$ & $5095-5105$ & $5620-5635$ & $6197-6201$ \\
$4044-4047$ & $4585-4589$ & $5245-5255$ & $5760-5766$ & $6335-6347$ \\
$4268-4279$ & $4740-4755$ & $5372-5381$ & $5990-5998$ & $6638-6643$ \\
$4420-4430$ & $4830-4840$ & $5515-5525$ & $6021-6025$ & $6771-6794$ \\
$4422-4428$ & $4965-4975$ & $5555-5565$ & $6057-6061$ & $6927-6933$ \\
\hline
\end{tabular}

into a global, high quality mean, which was then fitted in selected pseudo-continuum regions, i.e. wavelength regions where large emission-lines do not dominate. These regions are listed in Table 2. To avoid having to use high order polynomials for the fit of a very large wavelength region, we cut the spectra in 5 different sections to fit, i.e. $3999-4454 \AA, 4400-4794 \AA$, 4750-5294 A, 5229-6032 $\AA$ and 5999-7000 A. Finally, the fitted continuum function is applied to each individual spectrum. The error on the continuum normalization measured as the standard deviation of individual spectra around the continuum function is typically of $0.5 \%$.

\section{Results}

\subsection{Temporal variance spectrum}

In order to identify the variable parts of the spectrum of WR 123, we adopt the "temporal variance spectrum" (TVS) approach developed by Fullerton et al. (1996). To minimize the influence of intrinsic radial velocity (RV) variations of WR 123 on the TVS, we co-align the spectra using cross-correlation. All the spectra were resampled after the co-alignment at the resolution of the spectra obtained at NTT, since they are the most numerous in our dataset and they have the lowest spectral resolution. The resampling was made using a sinc function. This method tends to slightly broaden the line profiles (negligibly in the case of WR spectral lines), but it does not modify the level of noise in the spectra and, therefore, does not affect the TVS. We find that all the spectral lines in the observed spectral domain, $\lambda \lambda 4000-6940 \AA$, show lpvs significantly above the adopted 99\% threshold. All reasonably isolated spectral lines produce a similar shape in the normalized TVS plot. However, in a given P Cygni profile the absorption displays a single narrow peak in the normalized TVS spectrum, and the emission part shows a wider and more structured peak with stronger variability in the central parts (e.g., Fig. 2 with HeII $\lambda 5412 \AA$ and HeI $\lambda 5876 \AA$ as typical examples). This indicates that the detected lpv are not directly related to continuum variability. The latter would create a predictable global pattern with a shape following the emission-line contours.

\subsection{Line-profile and equivalent-width variability}

The TVS analysis shows that, in absolute terms, the most variable and [relatively] isolated lines are Her $\lambda$ 4471, 4922, 5016, 5876, $6678 \AA$; HeII $\lambda$ 4686, 4860, 5412, $6560 \AA$; and NiII $\lambda \lambda$ 4640, $41 \AA$.

To study the nightly changes in the line profiles, we single out two strong and least blended transitions, HeII $\lambda 5412 \AA$ and Her $\lambda 5876 \AA$. The most variable part of the Her $\lambda 5876 \AA$ line is its absorption component, though the emission also changes from night to night. No apparent recurrence or coherent behavior in the variability patterns can be found at first glance. The HeII $\lambda 5412 \AA$ line does not precisely follow the changes in Her $\lambda$ $5876 \AA$. However, the lines behave in a roughly similar manner, i.e. the amplitudes of the absorption and emission components vary strongly and seemingly randomly on a timescale of days and longer.

From inspection of the remaining lines we conclude that what determines the global variability patterns of a line on a timescale of days is the strength of the P Cygni absorption component. Indeed, regardless of their species, all lines with a relatively weak absorption component change similarly to HeII $\lambda$ $5412 \AA$ while all lines with a strong P Cygni absorption behave like Her $\lambda 5876 \AA$. 
On a timescale of hours all the lines show similar lpv in shape and in relative intensity, if one accounts for spectral blending effects. Within a given night, intensity and width can vary from $5 \%$ to $30 \%$, with the latter, if applied to strong emission features, in clear excess of the variability expected from the ever-changing continuum level on the same timescale.

In an attempt to quantify the changes for each of our selected lines, we examine their equivalent widths (EW). Since the variability of the absorption and emission components of P Cygni profiles do not appear to be correlated, we have treated their EW measurements separately by fitting two gaussian functions, one with a negative intensity and displaced from the line center (absorption) and another with a positive intensity (emission). The measurement errors were obtained following Vollmann \& Eversberg (2006). Though realizing that such an approach may not be applicable to all considered cases, we note that most of the 2-component fits have a reasonably low $\chi^{2}$ and produce fairly good results for the blue-side portions of profiles.

In an attempt to sample a significant part of the WR wind we choose the Her $\lambda 4471 \AA$ (which gives better fits than Her $\lambda$ $5876 \AA$ ) and HeII $\lambda 5412 \AA$ lines, both of which are strong, isolated and relatively easy to fit. We find that, in general, a stronger emission corresponds to a stronger absorption, and vice versa. However, for the HeII $\lambda 5412 \AA$ line, there are frequent disparities between the variability patterns of the emission and absorption components. Moreover, even if both lines (HeI and HeII) vary with comparable amplitudes on a timescale of days, their variability within one night can be completely different. Taking into consideration the rest of the measured lines, we find that the EW of the emission parts of the HeII, NIII and Niv profiles are well-correlated, while quite frequently the Her lines lack correlation with any other species and among themselves. This could be partially blamed on the presence of strong absorption components with a pronounced tendency to show different lpv patterns. The lack of correlation is especially prominent in the long-term (days-months). However, a tight HeI - HeII correlation may reappear in some of the short-term (nightly) data segments.

\subsection{Radial velocity and skewness variability}

Previous RV studies of WR 123 have consistently shown the presence of small-amplitude velocity variations with $K \leq$ $25 \mathrm{~km} \mathrm{~s}^{-1}$ (Marchenko et al. 1998). Unfortunately, the NTT and CFHT data produced large systematic errors related to specific instrumental setups. This leaves OHP as the only reliable source of RV. We aligned the OHP spectra via cross-correlation with the highest $\mathrm{S} / \mathrm{N}$ spectrum (the first spectrum of the campaign in this case), thus producing a mean OHP spectrum. Then we used this mean to perform a cross-correlation with individual spectra one more time and measure their RV. RVs were checked with the bisectors of the strongest emission lines. While the bisectors were giving bigger errors, they match the results coming from cross-correlation and show that all the lines are shifting in time with the same fashion.

On a weekly time-scale, the measured RVs vary by up to $\pm 20 \mathrm{~km} \mathrm{~s}^{-1}$, well in excess of the typical errors, $\sigma \sim 2-5 \mathrm{~km} \mathrm{~s}^{-1}$ (obtained from the fit of the cross-correlation profile), by gradually decreasing from $\sim 0 \mathrm{~km} \mathrm{~s}^{-1}$ to $\sim-40 \mathrm{~km} \mathrm{~s}^{-1}$ (with respect to the first spectrum of the campaign). A closer look shows that the RVs vary from night to night and can change by as much as $10 \mathrm{~km} \mathrm{~s}^{-1}$ within a $\sim 4-\mathrm{h}$ interval. Are these RV variations caused by the presence of a companion or simply by large-scale lpv? One possible way to verify this is to examine the skewness of the main spectral lines, i.e. HeIr $\lambda 5412 \AA$ and Her $\lambda 5876 \AA$. Since these two lines clearly dominate the spectrum (and therefore the flux in the cross-correlation profile) in the chosen wavelength range, they are also the main contributors to the RV measurements. We find that the skewness varies on a short timescale of $\sim \mathrm{h}$. Hence, the variability of the RVs may be induced by largescale, short-term profile variability, save the long-term downslope in the RV curve. It is not possible to relate any particular change in the overall line shape to a particular change in RV. With some caution, we conclude that the only "genuine", i.e., caused by a possible spatial (orbital?) motion of the star, RV variability may occur on a fairly long timescale of days or weeks.

\subsection{Photometric variations}

Our light curve (Fig. 1) has fairly similar characteristics to the light curve published by Lefèvre et al. (2005). Both have a variation amplitude of $\sim 15-20 \%$ on the long term and $\sim 5 \%$ on the short term. Hence, we can assume that nothing major in the star and the star's wind has changed between the 2003 spectroscopic campaign (this study) and the 2004 MOST campaign (Lefèvre et al. 2005) and that the two datasets can be compared. However, the limited photometric accuracy and the poor time sampling of our data compared with the unique MOST observations does not allow us to say anything more about WR 123 's photometric variability.

\section{Period search}

We performed a period search in the permittable range from 10 min up to one month, using two complementary approaches. The phase-dispersion minimization algorithm (PDM, Stellingwerf 1978) is well-suited for cases with only a small number of observations and highly non-sinusoidal underlying signal. We also used the CLEAN algorithm (Scargle 1982; Roberts et al. 1987), which has the advantage of taking into account the unevenness of the data sampling.

We searched for periodic signals in the RV data and found that the OHP subset reveals no significant periodicities. Period search on the nightly mean EWs of the HeII $\lambda 5412 \AA$ line provides inconclusive results, mainly due to the scarcity of these particular (1 point/night) data. Searching for short periods, we concentrated on our numerous NTT spectra, first carefully removing any long-term (days-weeks) trends. We find a period of $0.400 \pm 0.004\left(v=2.50 \pm 0.06 \mathrm{~d}^{-1}\right)$, which is compatible with the range of frequencies between $2.40 \mathrm{~d}^{-1}$ and $2.50 \mathrm{~d}^{-1}$ claimed by Lefèvre et al. (2005) from MOST data, is present in the de-trended EW measurements of both HeI (peak at $2.46 \mathrm{~d}^{-1}$ ) and HeII lines (peak at $2.51 \mathrm{~d}^{-1}$ ) and is clearly detected by PDM, but only marginally present in the cLEANed PS. The detrended EW values of HeII $\lambda 5412 \AA$ folded in phase with this period are shown in Fig. 3.

Since the amplitude of the photometric variations on the timescale of a few hours is relatively small compared with the accuracy of our photometric dataset and due to our poor time sampling, no clear period could be found in the light curve.

\section{Discussion}

Most of the lines in the WR 123 spectrum have a P Cygni profile, i.e. they contain a blue-shifted absorption component formed in the column of wind between the stellar core and the observer, as well as an emission component formed in a (more or less) 

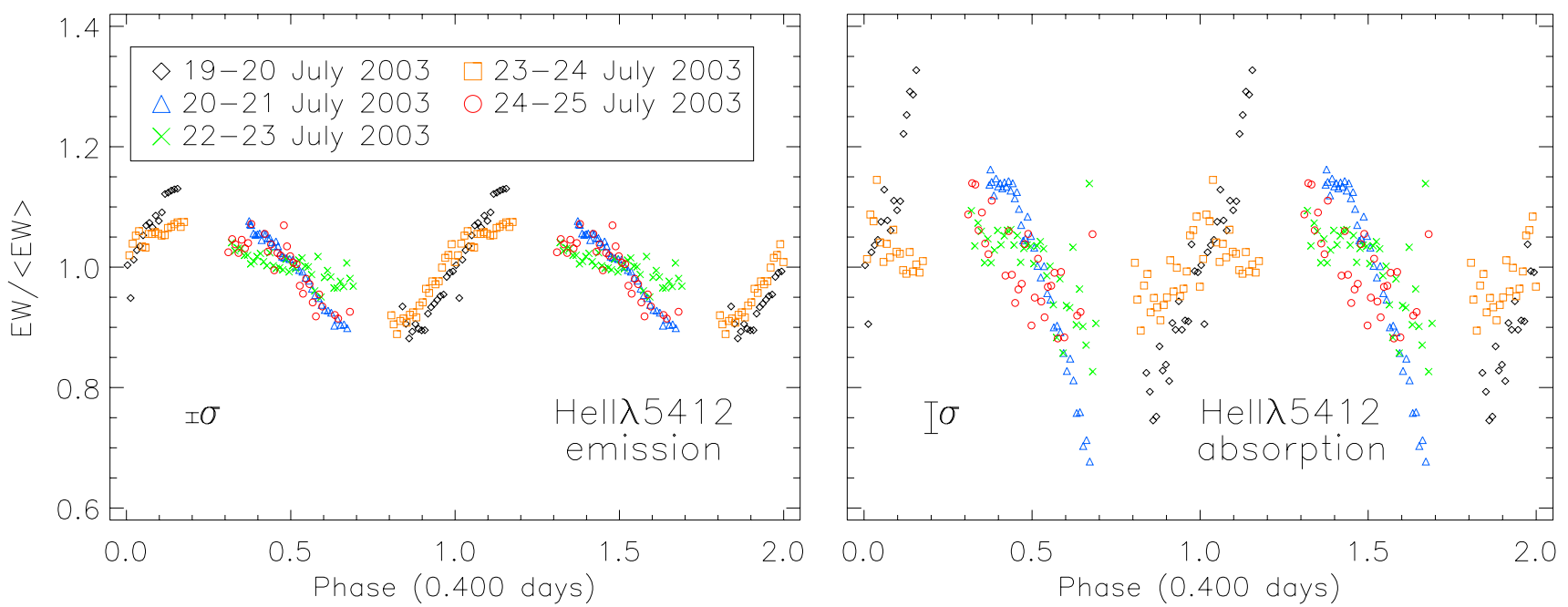

Fig. 3. The de-trended EW measurements of the emission component (left panel) and of the absorption component (right panel) of the HeII $\lambda 5412 \AA$ line folded in phase with $P=0 \mathrm{~d} .400$ and an arbitrary 0 phase. Five half-nights of the second NTT run are shown along with the corresponding $1 \sigma$ error bars.

symmetric shell surrounding the core. Since the two components produce different variability patterns, we treated them separately wherever feasible.

All the emission components vary with similar shapes and relative amplitudes, up to $30 \%$ (peak-to-valley) of an average line intensity on a short timescale (hours), and up to $55 \%$ on a long timescale (days). There is no delay between the variability patterns of the different lines; i.e., the variability seems to occur simultaneously in the different line-forming regions of the wind.

The absorptions have a seemingly higher level of variability, up to $50 \%$ of their depth on a short timescale, and as high as $90 \%$ on a long timescale. However, this difference may come from the intrinsic difference between the formation process and the different range of intensity of the absorption ( 1 to 0 relative intensity) and the emission ( 1 to $I$ relative intensity, where $I$ is the intensity at the peak) parts of P Cygni profiles. Moreover, the variations of the two parts of any given P Cygni profile have similar shapes, indicating that they can be caused by the same phenomenon. All absorption components vary in concert, without detectable delay between different line species. Considering that the star shows a consistent level of photometric variability that practically never exceeds $\sim 0.15$ mag long-term (daysmonth: e.g. Marchenko et al. 1998; Lefèvre et al. 2005, our study) and rarely exceeds $\sim 0.05$ mag during any particular night of ground-based observations (e.g., Fig. 1), such a level of lpv cannot be a consequence of the continuum variations alone. We note that for WR 123 the continuum flux is the dominating (usually up to $80 \%$ ) component in the broadband photometric optical flux.

In the following we concentrate on the 0.4 period found in the de-trended EW data. We plot the period-folded lpv of the strongest and least blended lines He iा $\lambda 5412 \AA$, Her $\lambda 5876 \AA$ and HeII $\lambda 6560 \AA$ in Fig. 4 . In the bottom parts of the panels we plot average profiles derived from four different phase intervals, $\phi=0.0-0.2, \phi=0.3-0.5, \phi=0.5-0.7, \phi=0.8-1.0$. It is apparent that practically the whole wind responds to the periodic perturbation coming from the core. In general, the absorption components are more involved in the periodic changes, as they come from a more localized region of the wind compared to the emissions. This does not hold true for HeI $\lambda 5876 \AA$ which has the largest line-forming region among the considered lines, thus
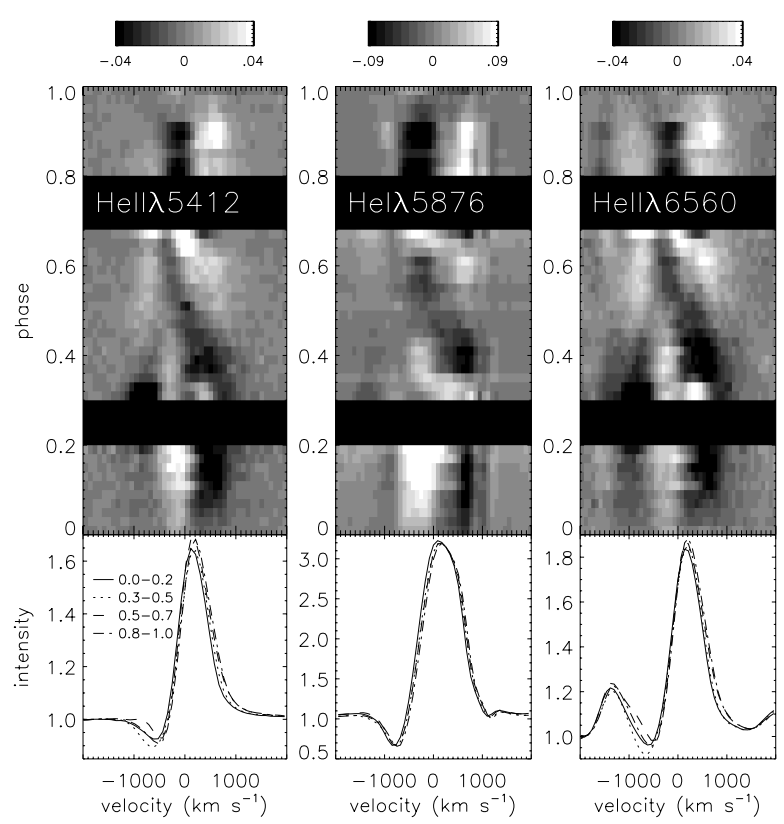

Fig. 4. Upper: Lpvs observed in the HeII $\lambda 5412 \AA$ (left), Нег $\lambda 5876 \AA$ (center) and HeIr $\lambda 6560 \AA$ (right) folded with the 0 d4-period. Longterm trends have been removed from the spectra and the remainders were enhanced by a factor of 2 for clarity. Lower: binned profiles over the four phase intervals $\phi=0.0-0.2, \phi=0.3-0.5, \phi=0.5-0.7$, $\phi=0.8-1.0$.

the best ability to "smooth" out the phase-dependent variability patterns.

Lefèvre et al. (2005) already dismissed the possibility that stellar rotation could be at the origin of the $9.8 \mathrm{~h}$ period, since this would lead to a rotational velocity greater than the breakup speed at the surface of a typical WN8 star (when taking $R_{*} \sim 15 R_{\odot}$ according to Crowther et al. 1995). Also, they showed that such a short period could not be produced by the presence of a (compact) companion revolving around the star, since the deduced orbit would be smaller than the stellar hydrostatic radius of WR 123 . Finally, they placed a firm upper limit, 
$A<0.2$ mmag for $\mathrm{P} \lesssim 1 \mathrm{~h}$, on the amplitudes of strange-mode pulsations predicted by Glatzel et al. (1999).

Since the publication of the MOST light curve, two independent models involving non-radial pulsations were proposed to explain the $9.8 \mathrm{~h}$ period. The first, by Townsend \& MacDonald (2006), examines the stability of $\ell=1$ and $\ell=2$ g-modes and finds that the $\kappa$-mechanism associated with a hot Fe-opacity bump within the star can be responsible for periods ranging between 3 and $12 \mathrm{~h}$. The second model proposed by Dorfi et al. (2006) contradicts the conclusion of Lefèvre et al. (2005) concerning strange-mode pulsations. Indeed, using an adequate stellar radius for WR 123, they showed that strange-modes with a period as high as $\sim 10 \mathrm{~h}$ can be excited. However, both models exploited stellar parameters beyond the usually adopted range: Townsend \& MacDonald (2006) used a stellar radius that is far too small for a WN8 star, and Dorfi et al. (2006) used a hydrogen abundance which is too high for WR 123. More recently, Glatzel (2008) followed the evolution of strange mode instabilities into the non-linear regime and showed that they generate consecutive shock waves which inflate the stellar envelope considerably and thus increase the pulsation periods, giving final periods that are consistent with that observed in WR 123. It remains unclear how these pulsations will affect the stellar wind (thus the windformed spectral lines) at different radii. Our data show that the 9.8-h spectral variability involves all prominent lines in the observed spectral region, thus globally affecting the wind.

Finally, on a speculative note inspired by the suggestion of Foellmi \& Moffat (2002), one might wonder if a Thorne-Żytkow-like scenario could be operating in WR123 and other WN8 stars and exciting stellar pulsations. Thorne-Żytkow Objects (TŻO, Thorne \& Zytkow 1977) are assumed to be red supergiants with a degenerate neutron core. They are formed via merger of a massive star and a remnant of a supernova explosion in a close binary system. Interestingly, a TŻO nature of WN8 stars would explain their relatively large stellar radii, high large space velocities, the avoidance of $\mathrm{OB}$ associations and the (current) dearth of binaries.

\section{Conclusions}

Summarizing the results of our intense, multi-site monitoring of the variable WR star WR 123 in 2003, we note that:

1. The star shows pronounced long-term, large-scale, seemingly stochastic spectral and photometric variability, broadly in line with all previous studies.
2. The only stable period which is clearly present in the EW of prominent spectral lines matches the $P=9.8$-h period found in the MOST photometry obtained one year later.

3. Practically all the observed wind volume is involved in the 10 -h periodic variations. Though the variability patterns may differ among the lines with different ionization potentials, all the lines vary without measurable phase delays. This points to the stellar core as sole driver of the periodic variability.

Acknowledgements. A.N.C. gratefully acknowledges support from the Chilean Centro de Astrofísica FONDAP No. 15010003 and the Chilean Centro de Excelencia en Astrofísica y Tecnologías Afines (CATA). N.S.L. and A.F.J.M. wish to thank the Natural Sciences and Engineering Research Council (NSERC) of Canada for financial support.

\section{References}

Antokhin, I., Bertrand, J., Lamontagne, R., Moffat, A. F. J., \& Matthews, J. 1995, AJ, 109, 817

Balona, L. A., Egan, J., \& Marang, F. 1989, MNRAS, 240, 103

Crowther, P. A., Hillier, D. J., \& Smith, L. J. 1995, A\&A, 293, 403

Dorfi, E. A., Gautschy, A., \& Saio, H. 2006, A\&A, 453, L35

Drissen, L., St-Louis, N., Moffat, A. F. J., \& Bastien, P. 1987, ApJ, 322, 888

Foellmi, C., \& Moffat, A. F. J. 2002, in Stellar Collisions, Mergers and their Consequences, ed. M. M. Shara, ASP Conf. Ser., 263, 123

Fullerton, A. W., Gies, D. R., \& Bolton, C. T. 1996, ApJS, 103, 475

Glatzel, W. 2008, Stability and Pulsations of Wolf-Rayet Stars, in HydrogenDeficient Stars, ed. A. Werner, \& T. Rauch, ASPC 391, Tübingen, 307

Glatzel, W., Kiriakidis, M., Chernigovskij, S., \& Fricke, K. J. 1999, MNRAS, 303,116

Lamontagne, R., \& Moffat, A. F. J. 1987, AJ, 94, 1008

Lamontagne, R., Moffat, A. F. J., \& Seggewiss, W. 1983, ApJ, 269, 596

Lefèvre, L., Marchenko, S. V., Moffat, A. F. J., et al. 2005, ApJ, 634, L109

Marchenko, S. V., Moffat, A. F. J., Lamontagne, R., \& Tovmassian, G. H. 1996, ApJ, 461, 386

Marchenko, S. V., Moffat, A. F. J., Eversberg, T., et al. 1998, MNRAS, 294, 642

Massey, P., \& Conti, P. 1980, ApJ, 242, 638

Moffat, A. F. J. 1989, ApJ, 347, 373

Moffat, A. F. J., \& Isserstedt, J. 1980, A\&A, 91, 147

Moffat, A. F. J., \& Shara, M. M. 1986, AJ, 92, 952

Moffat, A. F. J., Marchenko, S. V., Seggewiss, W., et al. 1998, A\&A, 331, 949

Robert, C. 1992, Ph.D. Thesis, Université de Montréal, Canada

Robert, C., Moffat, A. F. J., Bastien, P., Drissen, L., \& St-Louis, N. 1989, ApJ, 347,1034

Roberts, D. H., Lehar, J., \& Dreher, J. W. 1987, AJ, 93, 968

Scargle, J. D. 1982, ApJ, 263, 835

St-Louis, N., Moffat, A. F. J., Drissen, L., Bastien, P., \& Robert, C. 1988, ApJ, 330,286

Stellingwerf, R. F. 1978, ApJ, 224, 953

Thorne, K. S., \& Zytkow, A. N. 1977, ApJ, 212, 832

Townsend, R. H. D., \& MacDonald, J. 2006, MNRAS, 368, L57

Vollmann, K., \& Eversberg, T. 2006, Astron. Nachr., 327, 862 